\title{
VERSITA
}

10.2478/v10284-012-0012-0

\section{REFLECTIVE PRACTISE AT PRAGUE COLLEGE}

\author{
JEFFREY BUEHLER
}

Prague College aspired in its first tentative steps into higher and further education to create the right conditions for learning by providing the required resources, qualified academic faculty, and sound administrative systems. Over its short existence the college did in this way achieve a certain level of stability and experience as an institution. Nevertheless, despite achieving some success at providing students with an education, it became less clear why the same problems consistently cropped up, in repeating patterns that confounded the many patchwork solutions provided.

The aim of this paper is to summarise the work done in the dissertation Assessing the Potential for Reflective practise within a College-wide Professional Development Strategy (Buehler, 2011). Based on this research, the paper will explore how the Prague College faculty, and in particular its leaders, would view reflective practise as a tool for self and organisational learning, and what its role might be within a new strategy for professional development. The research drew on both articulated and observed communication as it sought to answer the following questions in relation to the topic:

1. To what degree is reflective practise already actively present in the college systems and processes?

2. How is it currently understood and practised by faculty at the college?

3. What are the attitudes of the faculty towards the introduction of reflective practise?

4. What is most needed in terms of formal reflective practise?

5. What are the leadership and management considerations, including national cultural, that hinge on effective implementation of a new professional development strategy, and how might these considerations inform the strategy? 


\subsection{PRAGUE COLLEGE 'EXPERIENCE'}

The idea for the research arose in relation to personal inquiry into questions such as: why would dissatisfaction among administration or faculty come bubbling unexpectedly to the surface? Why did certain complicated situations, as well as some seemingly simple situations cause paralysis and consternation? It had become apparent that despite gaining ' experience' as an organisation, the college had not always learned as one. In other words, the experience gained had not always translated into learning. Accumulated knowledge would sometimes suddenly evaporate as change took place, shifting the overall balance of operations in a new, unexpected or alien direction. The resulting response would again be clumsy and ineffective, and basic lessons would need to be learnt all over again, as the fundamental norms and practises of the college remained unchanged, leaving the college incapable of reacting well to the next crisis or change (Argris and Schön, 1974).

The culture of the college could be seen through a prism of varied value systems (Coleman, 2004) and formed a colourful tapestry to work within. Handy differentiates between four typical organizational cultures (Handy and Aitken, 1986 in Coleman, 2004) and on the surface Prague College appeared to be a "Task Culture", with its varied and nuanced needs, requiring the flexibility to respond. However Prague College arose out of a potent and diverse mix of influences. Under these conditions without a clear understanding of, and confidence in, our values and principles as leaders, we had little chance to become an effective school, or to create an effective learning environment. As Anne Gold has remarked, "clearly articulated values leave a leader less likely to vacillate between different political and educational orthodoxies and more able to respond thoughtfully"(2004, p. 6). In my experience at the college in those early days, decision-making vacillated according to situations. In the general confusion and energy of setting up a new institution the leadership was often left awash in the powerful flow of external demands, and decisions were too often made not out of internal value systems, but out of expediency. Or, as Gold puts it, there lacked "... a calm or stillness at the centre of this pressure, so that sound decisions are made" (2004, p. 8).

In order to better manage this growing and changing environment, and in order to compete with the local environment, a strategy was established to develop a core Prague College faculty that would be unique to the school. The college had previously only hired lecturers on per module contracts, which allowed quicker response to circumstances as they changed, and also allowed more flexibility in designing a long-term faculty. However, the effect on the culture of the college was clear. In the first years there was no focus on research and little involvement with the development of the college beyond the modules lecturers were teaching. The short-term financial and organisational benefit achieved by this first strategy quickly became a long-term obstacle to improving as a school. In those first years the turnover of teaching staff was nearly 40 percent a year, and student satisfaction, based on feedback from the students, was low, and in some programmes dire.

The original vision of the Director at this stage was important, and included the idea that the college was to be a more integrated, interdisciplinary learning environment, student facing, a place where research and reflection underpinned practise. Over time, with increased confidence and finance a new strategy of faculty development emerged, and its primary purpose was to build a full-time faculty that was involved, present and actively seeking opportunities to develop themselves and the college. This also included the present leadership organization hierarchy.

This new strategy had the effect of enhancing the student experience at the college while providing an identity as an institution. Faculty turnover dropped to about $2 \%$ a year on a new teaching staff of around 40 . And student satisfaction, based on feedback and decreased attrition, rose steadily. Despite the substantial increase in overall cost, it was believed by senior management that this new strategy provided a long-term competitive advantage. The current view was that, over time, with the student and learning experience at the centre of concern, the reputation of the college would help to increase student numbers. Indeed, student numbers doubled or nearly doubled four years in a row. Unfortunately, however, the resulting increase in student 
numbers did not increase the capacity to understand and work with the additional forces that naturally came into play, including micro-politics, rivalries, personal struggles, and conflicting values, the college's role in the local society and in the lives of the students, who come from over 70 countries to live, study and work in the Czech Republic.

As Fullan says, "Leading schools requires principles with the courage and capacity to build new cultures based on trusting relationships and a culture of disciplined inquiry and action" (2003, p. 45). Building a new culture meant building a full-time faculty with the added moral responsibility for the personal and professional development of all members of the college. The Director, Head of Teaching, and Heads of the Schools formed a rough professional development strategy that included everything from teacher induction to full degree programmes. The college began to offer semi-regular one-off professional seminars developed by lecturers to improve their practise, the content of which was often developed and delivered by faculty members. The response to change and growth was to work with and enhance the teams and people currently involved in the college, while nurturing relationships with individuals as they came.

However, this professional development strategy primarily fell into the realm of technical-rationality as described by Schön (1983), the main focus being on the passing on of information and skills, based on the mainly rational observation of facts. And the ad-hoc nature of the delivery and topics did not address the deeper issues the college was facing (Fullan, 2007). In the end, though useful, the current strategy has not led to the kind of learning, both institutional or individual, that supports and allows individuals to experience and learn more easily from a changing and unpredictable environment. In addition, the responses to situations that arose within the college were often reactive, impulsive and served only to "fix"the immediate problem. Learning in this case is "single-loop" and does nothing to change the underlying norms, policies or practises that created the problem (Schön and Argris, 1974). What was going to help the college get beyond an ad-hoc, superficial learning experience and allow for the deeper consideration of underlying values and conceptions?

\subsection{REFLECTIVE PRACTISE}

Dewey placed reflection at the centre of learning from our everyday experience of the world and this notion has carried on in the work of other experiential theorists (1933). For example, "reflection and observation" is one of the four elements of Kolb's famous model of experiential learning (1975). In addition, through my personal studies and experience, it also became clear to me that one of the crucial and central aspects of my learning has been the ability to reflect on my experience, both as it is happening and later. After discussing this topic with the Director and other colleagues in the college, as well as observing the environment over the past six years in relation to the continuing professional development of the faculty and its strategy, it became clear that there was a gap in the learning process as an institution, and a focus on reflective practise could begin to address this gap. However it has also been pointed out in the literature that in order for true reflective practise to take root in an institution, there first needs to be a trusting, open and caring environment in which practitioners feel confident and willing to expose themselves in order to learn and improve their practise as professionals (Senge, 1990; Smyth, 2003; Osterman and Kottkamp, 1993).

The research presented in the dissertation was chiefly phenomenological in design, as it set out to explore the multi-faceted feelings and attitudes of the lecturing staff, and in particular their leaders, about whether the college is in fact such a place. Could we open ourselves to each other for the purpose of learning? Did the leadership of the college have an accurate understanding of the needs of the lectures in order to implement a strategy that would be effective and not be seen as autocratic, dogmatic or simply awkward?

The dissertation explored the actual situation through a survey, interviews and observations, in order to capture and distil individuals' experiences, emotions, and insights with the aim to develop a strategy that took into account a broader understanding of how learning could take place most effectively at Prague College. 
At the heart of the learning process is the ability to reflect (Dewey, 1997; Kolb er Fry, 1975; Boud, 1985; Senge, 1990), and the review of the literature examined the practise of reflection and its role in the learning process, and how learning also takes place at the institutional level.

\subsection{REFLECTIVE PRACTISE: THE INDIVIDUAL LEARNING}

Dewey is often cited as one of the first to articulate and define 'reflection' in the context of learning, and his understanding of thought and reflection run through the work of future key writers such as Boud (1985) and Schön (1983; 1987). In particular Dewey's book How We Think provides a good starting point for describing the history of reflective practise. He defines reflection as "active, persistent, and careful consideration of any belief or supposed form of knowledge in the light of the grounds that support it and the further conclusions to which it tends"(1997, p. 6). The practitioner is asked to actively ' consider' any belief or the understanding of any other apparent phenomenon based on present evidence. The result of such an inquiry has the potential to bring new understanding. Of interest in the dissertation was the implementation of the conditions in which a practitioner may most effectively be allowed to actively, persistently and carefully ' consider'.

David Kolb and Roger Fry (1975) later explore effective learning and argue that there existed four different abilities: concrete experience abilities, reflective observation abilities, abstract conceptualisation abilities and active experimentation abilities. Kolb developed these into both a set of learning styles and his influential "learning circle" (1984). Kolb used models based on theories by Dewey, Lewin, and Piaget to describe learning as a continuous process that is grounded in direct experience, that "knowledge is continuously derived from and tested out in the experience of the learner" (1984, p. 27). Kolb's work in this area provided a crucial link to reflective practise as described by Schön (1983), who went on to provide the context in which professionals could learn to "reflect-in-action" and learn on the spot through their direct experience with situations.

In reflective practise, observable behaviour, which includes decisions and how they are made, habits, and actions, is governed by personal action theories (Osterman e Kottkamp, 1993). Theories in this case refer to the ideas that people attach to phenomena to explain how things works. These theories are associated closely with the personal experiences and everyday life of the practitioner. Using the notion of personal action theories, Schön and Argyris describe (1974) in great detail the difference between "espoused theories" and "theories-in-use". Espoused theories are essentially the views, values and beliefs that professionals believe their actions are based on, and will typically describe this beliefs to others. Theories-in-use are the views, values and beliefs that are demonstrated by their actual behaviour or actions. These behaviours are the observable consequence of the deeply imbedded maps, or patterns, that people carry in their minds, and which they make use of when taking action or making a decision (Argyris and Schön, 1974). In addition, people are often unaware that their theories-in-use are different from their espoused theories, and they can be completely oblivious to the existence of their actual theories-in-use (Argyris and Schön, 1974). This can result in situations, for example, where a school director of a private university states that academic standards are more important than short-term earnings. However when it comes to deciding on an individual student she may subtly manipulate situations in order to keep students in the school who either cannot cope or have failed to demonstrate the ability to perform, with the motivation being not to help the student but to keep them paying tuition. She may be completely unaware of the conflicting espoused view and the practise, and she may employ a number of defensive routines to protect herself and others from this knowledge (Argyris, 1994). 
A practitioner may work to bring theories-in-use and espoused theories in line, and in this process become more effective (Argyris, 1980). According to Senge (1990) the practise of reflection and inquiry is vital in this process of bringing the two together. Senge refers to theories-in-use as "mental models" in his book The Fifth Discipline, stating that what is most important to grasp is that these models are "active" and that they "shape how we act" (p. 164). And that the "problem with mental models is that they exist below the level of our awareness" (p. 166). He argues, based on the previous work of Argyris, that the most effective way to examine and expose mental models is through the reflective process of inquiry and advocacy. As mental models can act to thwart change, "managers must learn to reflect on their current mental models - until prevailing assumptions are brought out into the open, there is no reason to expect mental models to change..." (p. 189). Senge stresses that by opening ourselves, our beliefs and ideas to public scrutiny an atmosphere of genuine vulnerability is created, and for this reason combining inquiry and advocacy in a highly political organisation that is not open to genuine inquiry can be challenging (1990). This means that any development strategy that calls for reflective practise would need to take into account the context in which it happens, and understand the vulnerability that practitioners would feel when asked to expose their own "mental models" or theories-in-use, potentially even public scrutiny, as suggested by Senge (1990).

\subsection{THE REFLECTIVE PRACTITIONER}

Schön in his seminal book The Reflective Practitioner draws a clear distinction between what he calls Technical Rationality, or the overly formulaic application of knowledge and learning to situations that are by their very nature uncertain, unique and unstable - and the "epistemology of practise implicit in the artistic, intuitive processes which some practitioners do bring to situations of uncertainty, instability, uniqueness, and value conflict"(1983, p. 49). How does this happen? The practitioner:

allows himself to experience surprise, puzzlement, or confusion in a situation which he finds uncertain or unique. He reflects on the phenomenon before him, and on the prior understandings which have been implicit in his behaviour. He carries out an experiment which serves to generate both a new understanding of the phenomenon and a change in the situation. (Schön, 1983, p. 68)

When a professional is acting, carrying out the craft of her profession as described above, Schön calls this "knowing-in-action". This is the tacit, intuitive knowledge or feeling for situations, materials or phenomena which are often just beyond words. A bowler for example may not be able to describe precisely what he ' knows' as he moves his body through space to send the bowling ball on its way to a strike. There is a knowing that happens as the body and mind respond to moment-to-moment situations, variables and relationships that arise as typically uncertain, unstable and unique phenomena.

Applied to an organisational environment, often professionals are unable to effectively respond by applying certain positivist epistemologies of practise (Schön, 1983). Such knowledge and practises cannot hope to address the uniqueness of the phenomena with which professionals grapple. Rather, what happens in practise is that they respond through their experience of knowing the "feel" of the situation (Schön, 1983).Professionals may also begin to actively think about their actions as they are performing them, and consequently inquire more deeply into them, probing the tacit knowing-in-action for limitations, discrepancies and gaps in knowledge. This active inquiry into knowing-in-action is how reflection-in-action takes place (Schön, 1983), and echoes Dewey's "active, persistent and careful consideration" (1997, p. 6).However difficult it may be to articulate intuitive knowledge, the practitioner, with the right circumstances and support, can still investigate the situation, asking, ' why has this happened in this way?' ' What are the reasons for this behaviour and what is my role in it?' It is possible that this happens on the spot, based in concrete experience, in an experimental way, in much the same way that Kolb describes the experiential learning circle, with its elements of experience, reflection and experimentation (1984). 


\subsection{ORGANISATIONAL LEARNING: CREATING THE CONDITIONS FOR AN ENVIRONMENT OF REFLECTION}

It could be concluded from this that in order for reflection to take place as described, there first needs to be an environment which safely allows practitioners to experience "surprise, puzzlement or confusion" (Schön, 1983, p. 68). An environment which gives space for reflection on unexpected phenomenon and situations and which allows or even encourages experimentation and gives the practitioner that freedom to make changes based on the new insight. Unfortunately, however, Schön, does not describe the greater environmental factors required for effective reflection-in-action to take place. With the exception of his work with Argyris on single-loop and double-loop learning (1974) Schön tends to focus more on the individual processes of reflection and learning.

So what does reflective practise mean in terms of organisational learning, if we understand that the practise is a way to examine, expose or consider, the deeper patterns, or models, on which individual actions are based? As mentioned above, Argyris (1974) described two levels of learning that can take place in an organisation: single-loop learning and double-loop learning. Single-loop learning is the detection and correction of error that moves an organisation towards the expected outcomes and away from the sidetracked or errant outcomes. Double-loop learning involves the questioning of underlying policies, goals or norms of the organisation itself, with the intention of fundamentally altering the conditions in which the error took place (Argyris, 1977). For example a new strategy of professional development might require teachers to complete a course in time management because there is a problem in the school with teachers not having enough time. This would be an example of single-loop learning, whether or not this course improves the situation in the school. Double-loop learning would involve the examination of the underlying reasons of why teachers might be too busy and look to change the causes in the prevailing norms of the organisation.

\section{WHAT WAS DISCOVERED}

The following section lays out the findings under each of the original research questions using evidence collected from the methods previously described.

\section{TO WHAT DEGREE IS REFLECTIVE PRACTISE ALREADY ACTIVELY PRESENT IN THE COLLEGE SYSTEMS AND PROCESSES?}

The survey questions employed for this were meant to identify the extent to which the conditions at the college are already supportive of reflective practise, if there is a degree of openness and trust (Osterman and Kottkamp, 1993, p. 44).

If the 19 respondents have observed colleagues looking for systematic causes of problems, and if they feel that the college already asks them to reflect and they have observed others engaging in activity that encouraged reflection then one could partially gauge the degree to which reflective practise is taking place in the college systems.

The results in the survey indicated that the sample considered the college environment to be moderately, but not overly, open. The systems may encourage reflection to some degree, but the results expressed doubts and not a great deal of confidence in the way things were being done. The sample felt somewhat confident in its ability to reflect and for the systems at the college to allow for it. The standard deviation in this grouping was generally higher than in the other groupings, indicating a wider range of feelings in this area (Buehler, 2011). 
In response to the open question of what would you change about the college in order to create a more open and supportive environment for lecturers to work, all of the many comments were constructive and several noted that the college was already ' open and supportive'. The most useful comment in relation to the study was L.1 who suggested that the college:

create a more systematic way of allowing the teaching staff to develop. More structured approach within the college to allow this to happen rather than the ad hoc approach used at the moment where some teachers benefit as they are interested in this whilst others who feel they have nothing to learn, flat-line as professionals. L.1

The ad hoc approach mentioned describes succinctly what many of the comments hint at, and is also reflected in the comments of the leaders in the interviews. The college current development practises lack a systematic approach, which could account for the lower results above, despite having a generally caring and supportive environment. This demonstrates a need for a more comprehensive strategy that encourages more than just the interested parties to participate.

All eight interviewed leaders in the college described the college learning environment as being positive in many ways, with comments ranging from "the best I have ever worked in" to "it's open, with a lot of contact" and "it's very easy going, sort of calm."

Leader 1 expressed it this way:

I think that one of the main advantages of the college is the type of organisation that it is; it's quite flat, with very high communication among different departments. So this helps a lot in discussing with all the different departments. To develop Interesting collaborations, which is excellent for students. In this respect I think the college is distinct from those environments which emphasize a lot of specialisations.

However, according to Leader 1, the feeling is also that there is little structure in the college to support reflection in the college's systems. It is felt that the potential is there but the support and mechanisms are not in place to allow it to take place.

In summary, there appears already to be a positive learning environment at the college, as described by many of the leaders and in the short answers in the survey. And this learning environment allows for practises like co-teaching to take place, which encourages reflective practise. However, the systems that are in place are ad hoc and not systematic, leading to a range of responses on different areas. For example formal feedback sessions to lecturers happen in some cases, but not all, and when they happen they are not particularly reflective in nature.

HOW IS REFLECTIVE PRACTISE CURRENTLY UNDERSTOOD AND PRACTISED BY FACULTY? This survey grouping took a look at how current faculty understand and practise reflection at the college, and if they thought that this was important as professionals (for example, getting feedback from colleagues or students or learning from their colleagues). For comparison it also asked for feelings concerning more traditional methods of learning and whether they thought that they were effective. 
The overall positive grouping indicated that lecturers saw the various current ways of practicing reflection in a positive light. Of note was the interest shown by most in looking into conflict rather than avoiding it. This, according to Senge (1990, p. 171), is a sign of a healthy organisation that was not suffering 'the basic diseases of the hierarchy" with people who are willing to face any situation at hand, and showed understanding of the potential power of the practise for the college (Buehler, 2011).

In the open answer format respondents were meant to give examples of what reflection meant to them as professional teachers. About 30\% of the 19 respondents simply listed techniques like getting and receiving feedback, dialogue, etc. But some presented insightful indications of how some teachers are considering their practise both within the college systems and outside them.

The emphasis in several other examples was on the experience of teaching outside of any formal processes, procedures or systems.

For me reflection is part of my life, it is a
process that is essential for an examined
life and hence needs to be part of the
living process. In teaching and lecturing
the reflective process is continuous and
post delivery. During the lecture the
continuous feedback in various forms from
the students forms part of the reflective
process in improving the delivery of
content to students. Post delivery (after
lectures) the reflective process is relating
to the reflection of events in the lecture
and increasing awareness. Leader 3

In this case no mention is made of a structured activity. What is important to the lecturer is the ' continuous feedback', which the lecturer uses to form a dialogue with the teaching situation in order to improve his or her practise. Other less nuanced answers see reflection as "constantly adapting my classes to the students' needs" (Leader 4) and "CONSTANTLY trying to think of better ways to develop classes to meet the needs of our students as well as the other lecturers" (Leader 5). These examples show a wiliness to engage in active improvement but less awareness of the ongoing reflective conversation with the situation (Schön, 1983).

Osterman er Kottkamp claim (1993), based on the work of Argyris, that avoidance is often due to the wish to maintain control and power. However, considering the strong community feeling in the college, as described in many of the interviews, it is more likely that members of the college do not wish to 'stir the pot.' That maintaining our collegial relationships and the peace of the community overrides the need to learn and grow. This is a different organizational defensive routine that was observed and also described by several leaders in the college and is also one that Argyris has explored in the literature (1999).

The overall evidence suggested that there was already an ingrained understanding and practise of reflection in the college, and there appeared to be an appreciation of reflection in its various forms, and an understanding of the need to reflect. However the current understanding of the practise may not be transferable, was not systematic or fully capable of skillfully being applied in interactions with others. 


\title{
WHAT ARE THE ATTITUDES OF THE FACULTY TOWARDS THE INTRODUCTION OF REFLECTIVE PRACTISE?
}

This grouping captured the positions of lecturers and their attitudes towards different formal reflective practises that could be introduced in the future in the professional development strategy.

According to the results the sample saw themselves as professionals and were overwhelmingly in favour of experimenting with different reflective practises, including observing and learning from other lecturers, reflective appraisal schemes, and exploring enquiries into conflicts. There was some scepticism about appraisals that were not reflective in nature and simply assess lecturer performance (Buehler, 2011).

The majority of the interviewees were interested in creating a formal strategy that included regular seminars and reflective, even experimental learning opportunities. Leader 5 wishes to make these events contractual, formalise the process, meaning that lecturers would be required to attend a certain number of sessions. Leader 5 says:

\begin{abstract}
I think we need to move away from the whole area of trying to convince the person. I don't think we need to. We just say it's part of the process, and we formalise the process. It makes it much easier that way. Then you can focus on the delivery, which is individual, and you can't formalise that. Then you can focus on the person, the style of teaching, the personality, etc.
\end{abstract}

This would address the ad hoc problem nature in the way systems are currently managed but raises other concerns, for example in the School of Art and Design where the faculty were more in favour of less structure, and a more natural process.

In conclusion, the attitudes of all the voices in the college towards the introduction of reflective practise were positive, sometimes overwhelmingly so. However when it comes to how this is to be accomplished there are differences of opinion and the attitudes shift. The leaders in particular from the School of Art and Design advocated a more ' natural' approach, without a lot of unnecessary meetings or time wasted in sloppy agendas. The Business School leaders pushed for a more contractual and systematic approach that removed the need to convince others of the importance of the practise.

\section{WHAT IS MOST NEEDED IN TERMS OF FORMAL REFLECTIVE PRACTISE?}

In the survey this was mainly addressed through the use of the open answers questions and a checklist which offered lecturers an opportunity to rank the possible formal practises in order from 1 to 5 from the most needed and useful to the participant as a lecturer to the least useful and needed.

The survey asked respondents to rank five potential activities in which reflective practise could take place from most useful to least useful.

Once again lectures expressed the wish to meet, have dialogue, and explore difficult issues, though this survey did not specify if this was as a group or between two colleagues. Workshops and observations also scored highly, whereas appraisals and writing reports faired poorly; this could be because of the current demands and time-consuming nature of teaching at the college (Buehler, 2011). A limitation to this survey question may be that lecturers presumed that ' difficult issues' were not related to their own performance, difficult or stressful topics but rather difficult classroom management or organisational issues.

What are the leadership and management considerations, including national cultural, that hinge on effective implementation of a new professional development strategy, and how might these considerations inform the strategy? 
The broader implications of this research demanded a deeper look at and analysis of the environment at the college and the organisational systems and structures that would support a college-wide professional development strategy based on reflective practise. The research set out to open up territory previously closed to the senior managers for the purpose of reflecting on what is it we are doing, or not doing, as leaders. In this final section a number of recommendations are made for the successful implementation of the strategy based on the multitude of voices that made up this research.

\subsection{RECOMMENDATIONS}

According to the findings there is already a basis on which to form the strategy ("The environment at the college is open and supportive enough to allow me to consider trying reflective practises such as having reflective meetings in which the root of conflicts is explored." Mean =2.21). Prague College has a faculty that considers teaching a professional activity (" consider teaching a professional activity" Mean = 1.10), describes the learning environment as positive ("the best I have ever worked in" to "it's open, with a lot of contact"and "it's very easy going, sort of calm"), and wishes to look more deeply into the causes of dilemmas (" believe that it is important to openly inquire with others into difficult and conflictive situations in order to understand them more clearly." Mean = 1.52).

As noted in the literature (Osterman er Kottkamp, 1993; Senge, 1990) trust is the essential condition for reflective practise to take root and thrive, and the research suggests that there is an atmosphere of basic trust, which perhaps needs some cultivation. However the conditions overall are in place for reflective practise to be introduced on the individual level (" am willing to experiment with different methods for reflecting on my performance" Mean $=1.73$ ). Some practises in the college already encourage reflection and development. One example is the practise of co-teaching a unit or module, which is considered by those lecturers an excellent ' natural' way to reflect on their practise as a teacher. Other practises in the college, like giving formal feedback, are not as effective in terms of asking lecturers to reflect or addressing problem areas. On the organisational level lecturers described the systems as too ad hoc and sometimes without purpose ('More structured approach within the college to allow this to happen rather than the ad hoc approach used at the moment"Leader 1) (Buehler, 2011).

According to Miles and Ekholm (1985 in Coleman, 2004) in order for school improvement to take place there needs to be "a systematic, sustained effort aimed at change in learning conditions and other related internal conditions"(p. 48). Therefore it will be important to standardise and regularise the processes and systems that support professional development in the college. Without this any strategy that is implemented will not be effective, and will continue to be seen as ad hoc, irregular and inapplicable. To realise this a clear contractual relationship to professional development could be developed.

The challenge, however, is also to create the conditions for standardization to be adapted willingly, and to be owned by the faculty in the different schools. According to Hopkins (2002, in Coleman, 2004) the "adaptive" approach, and especially in the case of the different conditions in the Schools in the college, is more likely to be effective. Allowing the Schools to develop their own strategies according to their own needs in the school, with set objectives to be attained, is one way to do this. The level of formality, the nature of the techniques employed and the style of management in implementation should fall within the domain of the Schools in the college, as there are different approaches and understandings coming out of each. 
A generalised ' programme' of reflection at the college would be a positivist and linear conception of teacher education and could eliminate the key elements of learning to reflect-in-action as described by Schön (Richardson, 1990). Rather what is required is a culture in which individuals are compelled naturally to learn through "making new sense of uncertain, unique or conflicted situation of practise" (p. 39, in Clift, et al. 1990).

In order for reflection to take place the culture must safely allow practitioners to experience "surprise, puzzlement or confusion" (Schön, 1983, p. 68). This can happen in more or less formal settings (Osterman er Kottkamp, 1993) and some evidence in the interviews among leaders suggests a need in the School of Art and Design to develop a strategy along less formal lines, but other evidence suggests a common need to meet, discuss, and hold formal workshops and other formal techniques. In both cases of informal and formal practise the culture in the college must give space for reflection on unexpected phenomenon and situations, and allow or even encourage experimentation (Schön, 1983). Practitioners must have the freedom to make changes based on new insights, whether in informal discussions or workshop formats.

The leaders in the college as a whole demonstrated at times a natural aptitude at reflection and the ability to learn through reflection; however it was not always the case that they could apply this skill with others. The leaders, which include the senior managers, Heads of School, and Programme Leaders, should receive more specific workshops and training in reflective practise in order to learn how to apply the reflective skills more effectively and transfer them to others. This training can then be brought into the specific development of the strategies in the individual schools. According to Argyris (1977) double-loop learning involves the questioning of underlying policies, goals or norms of the organisation, with the intention of fundamentally altering the conditions in which errors take place. The leadership in their training in reflective practise with others could create a culture of organisational learning through group sessions in which organisational mental models are exposed, and discussed (Senge, 1990). In this way the college could experience a culture that supports and encourages double-loop learning, or the constant questioning of its norms and practises Argyris, 1977). For this to happen the leaders in the college will need the support and training in reflective practises by external experts in the field.

According to findings the management considerations of time, budgeting and resourcing are all important, and these needs will have to be addressed through careful planning and agreement with colleagues on what the college can manage to support. According to Gold (2004), however, it is not always necessary to spend a lot of money and time encouraging professional development. A school which "pays attention to learning and teaching for everyone within the school community buzzes with discussions and thought-provoking arguments, as well as planned formal learning opportunities"(Gold, 2004, p. 33).

All of the management considerations are connected to the values shared by the leaders and managers in the college. As was revealed in the interviews, going forward from here with a strategy will be difficult without a statement of common values that creates a sense of institutional purpose. According to Gold et al. school leaders are driven by "personal, moral and educational values and are able to articulate these with total conviction, creating a clear sense of institutional purpose and direction" (2003, p. 135). Therefore the key recommendation that must be accomplished before pursuing a new initiative is the development of a common set of values.

If the college is to allow for difference within the organisation, expect lecturers to look into their own experience for answers to questions, and engage in greater learning environment then this set of values will need to be developed as a team, and referred to in addressing the changes as they take place. It is the responsibility of the leadership in the college to "consider fundamental beliefs" in the pursuit of deep change (Smyth, 2003, p. 52), change that goes beyond single-loop learning, but change that fundamentally alters the policies and norms of the college, in a concerted effort to see more clearly the causes and conditions of persistent dilemmas. 


\section{REFERENCES}

Argyris, C. and Schön, D. (1974) Theory in practise. Increasing professional effectiveness. San Francisco: Jossey-Bass. Argyris, C. (1977) Double-loop Learning in Organisations, Harvard Business Review. (Sept.-Oct) pp. 115-125 Argyris, C. (1994) On Organisational Learning. Blackwell: Cambridge, Mass. USA. Boud, D. et al. (eds.) (1985) Reflection, turning experience into learning. London: Routledge Falmer.

Buehler, J. (2011) Assessing the Potential for reflective practise Within a College-wide Professional Development Strategy. Unpublished MA dissertation. University of London, Institute of Education.

Coleman, M. (2004) Organisations: power, structure and culture. Leading and Managing Educational Change and Improvement, University of London Press.

Coleman, M. (2004) Educational Improvement and Change. Leading and Managing Educational Change and Improvement. University of London Press.

Dewey, J. (1997) How We Think. Canada: Dover Publications, Inc.

Fullan, M. (2007) The New Meaning of Educational Change (4th ed.), New York: Teachers College, Columbia University.

Fullan, M. (2003) The Moral Imperative of School Leadership. Thousand Oaks, CA: Corwin Press, pp. 29-45.

Gold, A. (2004) Values and Leadership. Institute of Education, University of London.

Gold, A., at al. (2003) Principled Principles? Values-Driven Leadership: Evidence from ten case studies of ' outstanding' school leaders. Educational Management and Administration. 31 (2), pp. 127-37.

Kolb, D. A. (1984) Experiential Learning. Englewood Cliffs, NJ.: Prentice Hall.

Kolb. D. A. and Fry, R. (1975) Toward an applied theory of experiential learning in C. Cooper (ed.) Theories of Group Process. London: John Wiley.

Osterman, K. and Kottkamp, R. (1993) Reflective practise for Educators: Improving Schooling Through Professional Development. California: Corwin Press, Inc.

Richardson, V. (1990) The evolution of reflective teaching and teacher education, in Clift et al. (ed.) Encouraging Reflective practise in Education - An Analysis of Issues and Programs. New York er London: Teachers College Press.

Senge, P. (1990) The Fifth Discipline. New York: Doubleday.

Schön, D. (1987) Educating the Reflective Practitioner: Towards a New Design for Teaching and Learning in the Professions. San Francisco: Jossey-Bass Publishers.

Schön, D. (1983) The Reflective Practitioner. How professionals think in action. London: Temple Smith.

Schön, D. A. (1973) Beyond the Stable State. Public and private learning in a changing society. Harmondsworth: Penguin.

Smyth, R. (2003) Concepts of Change: Enhancing the practise of Academic Staff Development in Higher Education. International Journal for Academic Development, Vol. 8, No. 1/2. May/November. 